\title{
Shortened dental arch concept shown to be cost effective
}

\author{
Abstracted from
}

McKenna G, Allen F, Woods N, O'Mahony D, Cronin M, DaMata C, Normand C.

Cost-effectiveness of tooth replacement strategies for partially dentate elderly: a randomized controlled clinical trial. Community Dent Oral Epidemiol 2014; 42: 366-374. doi: 10.1111/cdoe.12085. Epub 2013 Nov 20. PubMed PMID: 24251386.

Address for correspondence: Gerald McKenna, Lecturer in Prosthodontics and Oral Rehabilitation, Cork University Dental School and Hospital, University College Cork, Cork, Ireland. E-mail: g.mckenna@ucc.ie

\section{Question: In older patients is a tooth replacement strategy based on the shortened dental arch concept (SDA) more cost effective than partial removable dental prostheses?}

\section{Design Randomised controlled trial}

Intervention All patients received treatment to render them dentally fit. Patients were randomly allocated to either the removable dental prostheses (RPD) or the shortened dental arch (SDA) group. Patients in the RDP group were restored to complete arches with RDP using cobaltchromium frameworks according to a standardised protocol. For the SDA group, patients were restored to a shortened arch of ten occluding pairs of natural and replacement teeth using resin-bonded bridgework (RBB). Outcome measure Treatment effect was measured using the change in oral health-related quality of life (OHrQOL). For each patient the costs of delivering treatment were recorded by a research nurse during the intervention period. Laboratory costs were recorded as part of normal hospital policy for all patients. All of the dental materials used were recorded and given a unit price. The cost of professional time per patient was estimated using the highest point of the salary scale for the Community Dental Service in Ireland.

Results One hundred and thirty-two patients were randomised; 65 to the RPD group and 67 to the SDA group. Ninety-two patients (69.7\%) completed the study (46 in RPD group; 46in SDA group). There was no difference in the success rates of the two treatments over the period. Five pieces of resin-bonded bridgework (RBB) debonded and were recemented over the 12-month period giving a success rate of $95.5 \%$ for the RBB. Four patients discontinued wearing their RPDs; all four RPDS were fitted in the lower arch and included bilateral free end saddles, a success rate of $95.9 \%$. Both RPD and SDA groups demonstrated statistically significant improvements in OHrQoL scores after 12 months.

The total cost of achieving the minimally important clinical difference (MID) in OHrQOL for an average patient in the RDP group was $€ 464$.64. For the SDA group, the cost of achieving the MID for an average patient was $€ 252.00$. The cost-effectiveness ratio was therefore 1:1.84 in favour of SDA treatment.

Conclusions With an increasingly ageing population, many patients will continue to benefit from removable prostheses to replace their missing natural teeth. From a purely economic standpoint, the results from this analysis suggest that the treatment of partially dentate older adults should be focused on functionally orientated treatment because it is simply more cost-effective.

\section{Commentary}

Healthcare resources are finite and how to allocate them equitably is always a problem. This issue has been magnified by the global economic downturn, and publicly funded dental services face increasing pressure to justify the cost-effectiveness of the services they provide. At the same time, there is an increasing number of dentate elderly patients requiring tooth replacement to provide a functional dentition into their old age. ${ }^{1}$ The importance, therefore, of a cost-effective treatment approach in this population has great significance.

This timely and well-conducted randomised controlled clinical trial sought to address these issues by examining the cost-effectiveness of rehabilitation using removable dental prosthesis (RDP) versus a 'functionally orientated' treatment based on the shorteneddental arch concept (SDA) using resin retained bridgework. Efforts were made to eliminate bias where possible through appropriate randomisation and blinding. The participant number is based on a power calculation, drop-outs are accounted for and an intention to treat analysis was used.

The authors used a patient-centred outcome measure (OHIP-14) to determine treatment effect from 'baseline' to six and 12 months, providing a measure of oral health-related quality of life for each treatment.

All treatment was provided by a single clinician with advanced postgraduate prosthodontic training. The use of a single clinician reduces variability in treatment, but possibly threatens generalisability of the findings as, in many settings, this treatment is usually provided by general practitioners. The study protocol required all participants to receive 'extensive' oral hygiene instruction, non-surgical periodontal treatment, extraction of 'hopeless prognosis' teeth, restoration of carious lesions and defective restoration replacement. It was unclear whether the 'baseline' OHIP-14 measure was taken before or after this initial treatment. If it was completed before, then we cannot say how much of the change in OHIP-14 scores is due to the tooth replacement strategy alone, compromising the study's internal validity. Furthermore, the authors did not report baseline values for whether each treatment arm contained patients with similar denture-wearing experience. Indeed, other than gender and age, there was no report of other subgroup analyses.

Treatment arm costs were comprehensively accounted for. The mean cost in each arm was calculated on the basis of material costs and professional time, including unscheduled appointments. The total, mean treatment cost for RDP was $€ 586.37$ compared with $€ 384.05$ for SDA. Mean OHIP-14 scores improved in both groups 
by 6.31 (RDP) and 7.62 (SDA) points after 12 months although the statistical and clinical significance of the higher SDA score is not clear. The authors used existing literature to determine that the minimally important difference (MID) in OHIP-14 is five scale points. $^{2}$ Therefore both groups achieved this MID. This led the authors to report a cost to achieve the MID of $€ 464.64$ (RDP) and $€ 252.00$ (SDA) and cost-effectiveness ratio of 1.84. The figures used to calculate the cost effectiveness in Table 3, however, do not match those in the text. Indeed, these figures cannot be found anywhere in the paper and would deliver a cost-effectiveness ratio of 1.89. All patients in the RDP group received a Cobalt-Chromium framework prosthesis, more costly than an acrylic equivalent and may not be representative of routine management across different oral healthcare provision settings. It is possible that the cost to achieve the MID with acrylic prostheses would be lower and more comparable with the SDA arm.

Overall, the authors make a compelling economic, if not clinical, case for the use of fixed bridgework to achieve a shortened dental arch in partially dentate elderly patients within a publicly funded dental service. They conclude by suggesting that, based on the economics, we should move towards functionally orientated treatment based on the shortened dental arch. That said, the authors accept that 'with an increasingly ageing population, many patients will continue to benefit from removable prostheses to replace their missing natural teeth'.

\section{Practice points}

- Achieving a shortened dental arch by replacing missing teeth using resin-retained bridgework was found to be more cost-effective than full rehabilitation with removable dental prostheses

- Further cost-effectiveness analyses in this area should consider longer follow-up periods and the inclusion of implant-supported treatments in the study design.

Colin Levey and Craig Dunbarb

${ }^{a}$ Division of Restorative Dentistry and ${ }^{b}$ Division of Oral Health Sciences , Dundee Dental Hospital and School,

Dundee, Scotland, UK

1. O'Sullivan I, Lader D, Beavan-Seymour C, Chenery V, Fuller E, Sadler K. 2011. Foundation report: Adult Dental Health Survey 2009 (Technical information). http:// www.hscic.gov.uk/pubs/dentalsurveyfullreport09. [Accessed 9th March 2015]

2. Locker $D$, Jokovic $A$, Clarke M. Assessing the responsiveness of measures of oral health-related quality of life. Community Dent Oral Epidemiol 2004; 32: 10-18

Evidence-Based Dentistry (2015) 16, 19-20. doi:10.1038/sj.ebd.6401080 